\title{
Efficient Design Techniques of Flash ADC for High Speed and Ultra Low Power Applications
}

\author{
Sujata S. Kamate ${ }^{1}$, H. P. Rajani ${ }^{2}$ and Vidyavati Mallaraddi ${ }^{3}$ \\ ${ }^{1}$ Department of Electronics \& Communication Engineering, Hirasugar \\ Institute of Technology, Nidasoshi, Karnataka, India \\ ${ }^{2}$ Department of Electronics \& Communication Engineering, \\ K. L. E's Dr. MSS CET, Belagavi, Karnataka, India \\ ${ }^{3}$ Department of Electronics \& Communication Engineering, K. L. E's Dr. \\ MSS CET, Belagavi, Karnataka, India
}

\begin{abstract}
Analog to Digital converters are the essential components of todays digital world. As these convert real time signal into its equivalent digital code, must be designed efficiently. This paper presents design of 3-bit high speed and power efficient Flash Analog to Digital Converter. Flash type Analog to Digital Converter is designed and implemented with different inverter based comparators and based ROM encoder. A high speed ROM encoder is designed to convert thermometer code to its equivalent binary code. The proposed research compares various inverter based comparators such as Threshold Inverter, Quantizer comparator, Single Inverter Comparator, Single Inverter Comparator with the reference voltage and LTE comparators, designed using cadence design tools with 180nm technological library. The simulation results show that Single Inverter Comparator consumes less power 5.647uW among TIQ and R-TIQ comparators. Single Inverter comparator with reference voltage consumes less power however with more delay. Here Flash ADCs are designed with various inverter based comparators and ROM encoder. The results show that TIQ comparator based ADCs consume power of 298.9uW, whereas ADC designed with Single Inverter Comparator consumes $459.8 \mathrm{uW}$ power.
\end{abstract}

\section{KEY WORDS: ITIQ COMPARATOR, ROM ENCODER, SINGLE INVERTER COMPARATOR, TIQ COMPARATOR.}

\section{INTRODUCTION}

Analog to digital converters are the critical components of digital communication system. Flash ADC is suitable for applications requiring very large bandwidths due to its high speed. But power consumption is more because of an array of comparators to achieve parallel operation. It is used in digital oscilloscopes, radar, high density disk drives, IoT applications and in communication systems. The power consumption of the Flash Analog to Digital converter has to be reduced in order to have efficient

\section{ARTICLE INFORMATION}

*Corresponding Author: sskamate.ece@hsit.ac.in

Received 10th Oct 2020 Accepted after revision 30th Dec 2020

Print ISSN: 0974-6455 Online ISSN: 2321-4007 CODEN: BBRCBA

Thomson Reuters ISI Web of Science Clarivate Analytics USA and Crossref Indexed Journal

\section{Clarivate
Analytics}

NAAS Journal Score 2020 (4.31)

A Society of Science and Nature Publication,

Bhopal India 2020. All rights reserved.

Online Contents Available at: http//www.bbrc.in/

Doi: $h$ ttp://dx.doi.org/10.21786/bbrc/13.13/20 communication system. The three main design parameters of ADCs are speed, power consumption and area. To achieve high speed and lower power consumption, ADCs are implemented in variety of architectures.

The Flash architecture enables lower latency, superior flexibility and lower metastability error rate than other high-speed, low-to medium resolution ADCs. Block diagram of the Flash ADC is depicted in Fig. 1 (Lavania, $Y$ et al., 2013). The three major components of Flash ADC are resistor ladder, comparator and an encoder. A resistor ladder generates reference voltages by voltage divider network. Each comparator compares the applied input voltage with reference voltages; if the applied input voltage is greater than reference voltage, comparator gives ' 1 ' as its output, otherwise ' 0 '. An encoder converts thermometer code to an equivalent digital code.

A Threshold Inverter Quantizer comparator proposed in (Yoo, J. et al., 2003), proposed that "the reference voltages are generated by varying the length and width of the

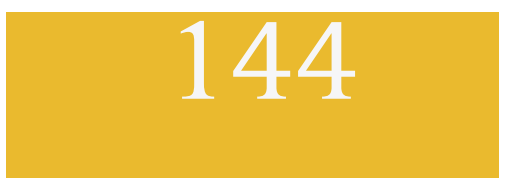


transistors as threshold voltage is the function width ' $W$ ' and length 'L'. TIQ comparator has two inverter circuits cascaded. The first one does comparing and the second balances. This circuit replaces the resistive ladder which consumes more area and results into more static power dissipation. The TIQ comparator is one of the high speed, area and power efficient techniques for designing Flash ADCs. The Linear Tunable Transconductance Element introduced in (Kulkarni, M. et al., 2010) reduces the power consumption by connecting two more transistors on either ends of the inverter circuit.

Figure 1: Block diagram of Flash Analog to Digital Converter

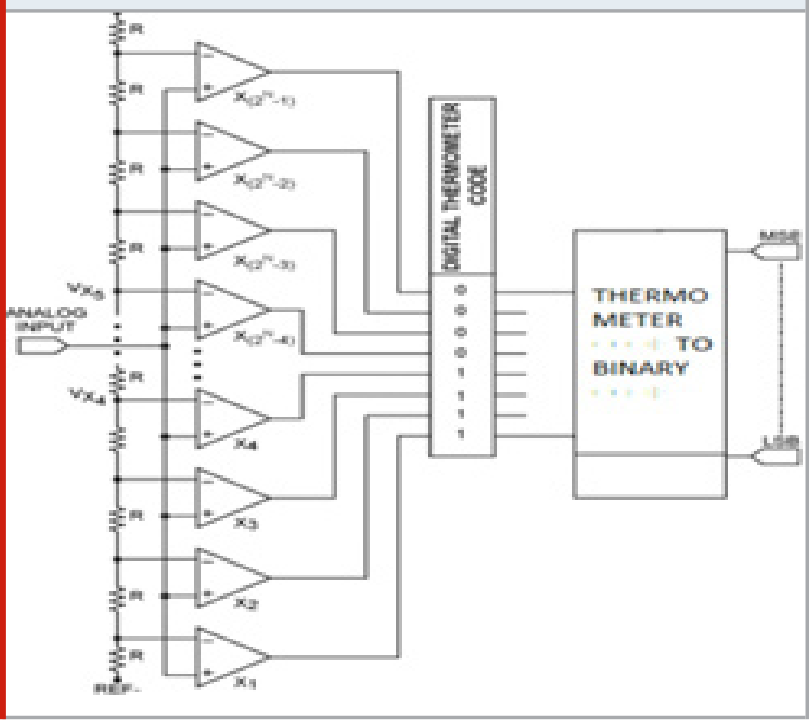

It shows a good improvement in power supply rejection ratio of the design but operates over the very little portion of the input signal swing. In (Gupta, Y. et al., 2014; Hosur, K.N. et al., 2015; Kar A. et al., 2015; Perumal, I. et al. 2009) proposed that Threshold Modified Comparator circuit operates over good range of input signal swing by varying 'W' and 'L' of TIQ comparator over a wide range, hence occupies more area. To enhance the range of signal swing Single Inverter Comparator with reference voltage is proposed in (Kalyani, N. et al., 2018). An improved TIQ comparator ITIQ with extra two pMOS transistors is proposed in (Prathiba, G. et al., 2018). A 3-bit Flash $\mathrm{ADC}$ is designed and analyzed with different inverter based comparators as they are power and area efficient circuits with high speed. The FADC design has seven comparators and 7:3 ROM based encoder. The paper compares performance of different inverter comparators and performance of FADC with each these comparators and ROM encoder.

Design of Comparators: The design of comparator is the most important part of the Flash ADC as the number of comparators doubles with 1-bit increase in the resolution of ADC. Power consumption of these comparators can be reduced by using different design techniques. This paper compares different parameters of Threshold Inverter Quantizer (Yoo, J. et al, 2003; Perumal, I. et al., 2009) Linear Tunable Transconductance Comparator (Kulkarni,
M. et al., 2010) and Single Inverter Comparators (Kalyani N. et al. 2018) and ITIQ proposed in (Prathiba, G. et al., 2018).

TIQ Comparator: TIQ Comparator has two inverters connected in series for comparing and balancing. By varying ' $W n$ ' and 'Wp' of the transistors the threshold voltage of the inverter circuits are changed to obtain different reference voltages. The Fig. 2 shows the designed TIQ comparator circuit which operates over $0.59 \mathrm{~V}$ to $0.930 \mathrm{~V}$ of input signal swing.

Figure 2: Threshold Inverter Quantizer Comparator

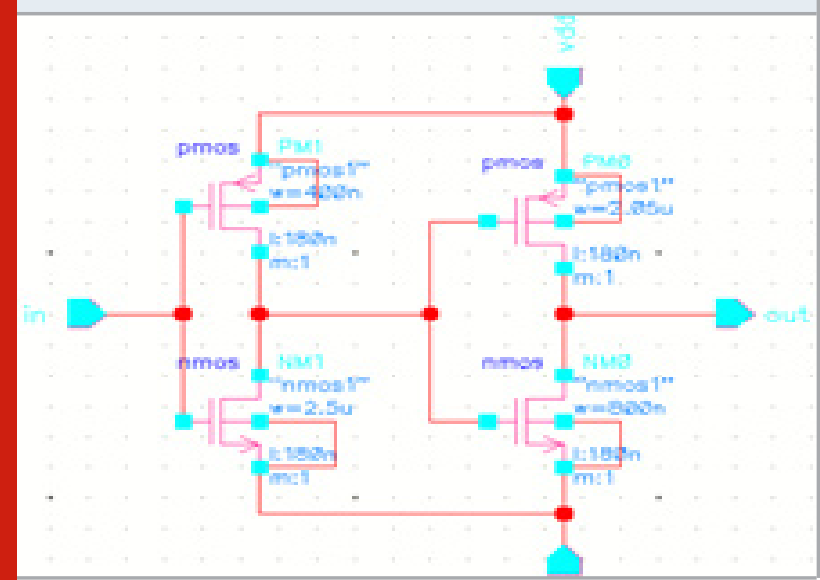

Equation 1 is used to obtain different threshold voltages of the inverters for various values of $\mathrm{Wp}$ and $\mathrm{Wn}$.

$$
V t h=\frac{V t n+\sqrt{\frac{1}{K r}(V D D+V t p)}}{1+\frac{1}{\sqrt{K r}}}
$$

Where,

process trnsconductance of nMOS is given by,

$$
K n=\text { un } \operatorname{Cox}\left(\frac{W}{L}\right)_{n}
$$

and of pMOS it is

$$
K p=u p \operatorname{Cox}\left(\frac{W}{L}\right)_{p}
$$

Tran conductance ratio $\mathrm{Kr}$ of the inverter circuit is given by,

$$
K r=\frac{K_{n}}{K_{p}}=\frac{u_{n} \operatorname{Cox}\left(\frac{W}{L}\right)_{n}}{u_{p} \operatorname{Cox}\left(\frac{W}{L}\right)_{p}}
$$

LTE Comparator: LTE-Linear Transconductance Element comparator structure resembles TIQ comparator with extra two transistors as shown in Fig. 3. The sleep transistors are operated in linear region to achieve linear 
transconductance operation. This relaxes the power bounds on inverter circuit reducing power consumption and hence improved Power Supply Rejection Ratio (PSRR). The operating range over input signal swing of this comparator is less. The operating range offered by this design is $0.866 \mathrm{~V}$ to $0.885 \mathrm{~V}$ of the input signal swing of $1.8 \mathrm{~V}$. The LTE comparator designed by varying $\mathrm{Wn}$ and $\mathrm{Wp}$ from 400nm to $2.5 \mathrm{um}$ in $180 \mathrm{~nm}$ technology with supply voltage $\mathrm{Vdd}$ equal to $1.8 \mathrm{~V}$, consumes an average power of $0.740 \mathrm{uW}$ with $\mathrm{Vg} 1=1.6 \mathrm{~V}$ and $\mathrm{Vg} 2=0.2 \mathrm{~V}$.

Figure 3: Linear Tunable Transconductance element comparator

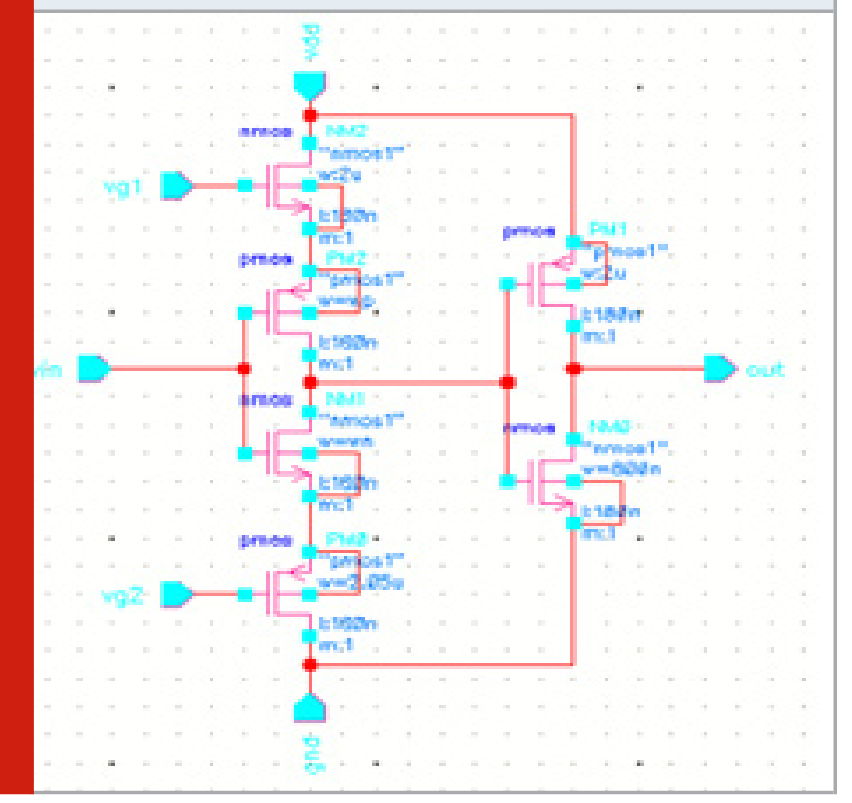

Figure 4: Single inverter comparator

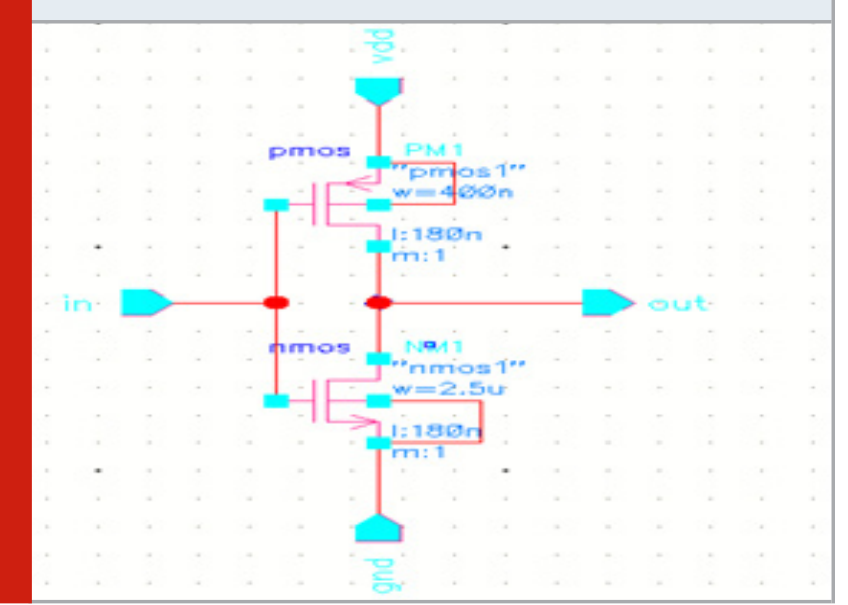

Single Inverter Comparator: The inverter circuit can be used for generating different reference voltages by varying its ' $\mathrm{Wp}$ ' and 'Wn' and comparing. The single inverter circuit generates the inverted outputs. The output is high when applied voltage is lesser than reference voltage generated by the inverter and becomes low when input is higher than reference voltage as in Fig. 4 shown below. This single inverter comparator operates from $0.615 \mathrm{~V}$ to $0.932 \mathrm{~V}$ of the input signal swing.

Single Inverter Comparator wit Reference Voltage: The above single inverter comparator operates almost over the entire input signal swing due to the reference voltage applied on the source terminal and reduces power dissipation by lifting the source voltage above ground. The threshold voltage values vary from $0.618 \mathrm{~V}$ to $1.315 \mathrm{~V}$ for Vref voltage values ranging from $0 \mathrm{~V}$ to 0.771 . The power consumption of this comparator varies with reference voltage; it is least for high reference voltages. The design with Vref $=0.771 \mathrm{~V}$ given in Fig. 5 consumes an average power of $2.94 \mathrm{pW}$.

Figure 5: Single inverter comparator with reference voltage

Figure 6: ITIQ comparator with reference voltage

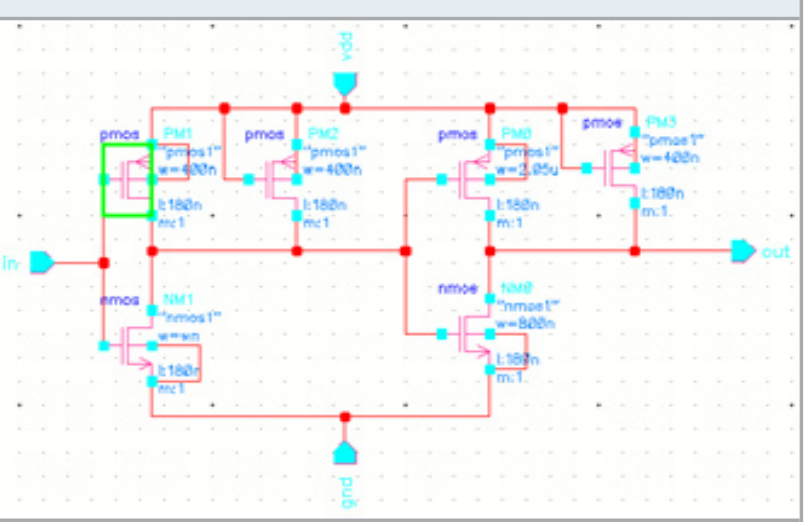

The above single inverter comparator operates almost over the entire input signal swing due to the reference voltage applied on the source terminal and reduces power dissipation by lifting the source voltage above ground. The threshold voltage values vary from $0.618 \mathrm{~V}$ to $1.315 \mathrm{~V}$ for Vref voltage values ranging from $0 \mathrm{~V}$ to 0.771 . The power consumption of this comparator varies with 
reference voltage; it is least for high reference voltages. The design with Vref $=0.771 \mathrm{~V}$ consumes an average power of $2.94 \mathrm{pW}$.

Improved TIQ Comparator: The improved TIQ comparator as in Fig. 6 has two extra p-transistors to get stable output of the comparator. These transistors will improve its performance and makes it more reliable. Table 1 gives the comparison between the various inverter based comparators. The simulation results show that single inverter and TIQ comparators are faster however with more power consumption. The LTE technique has better results but covers very little range of input signal swing.

\begin{tabular}{|c|c|c|c|}
\hline Comparator & $\begin{array}{l}\text { Input signal } \\
\text { swing(Vin) }\end{array}$ & $\begin{array}{l}\text { Avg. Power } \\
\text { Dissipation }\end{array}$ & Delay \\
\hline Single inverter & $0.615 \mathrm{~V}$ to $0.932 \mathrm{~V}$ & $5.647 \mathrm{uW}$ & 28.22usec \\
\hline LTE & $0.866 \mathrm{~V}$ to $0.885 \mathrm{~V}$ & $0.740 \mathrm{uW}$ & 1.38usec \\
\hline $\begin{array}{l}\text { Single Inverter with } \\
\text { (Vref }=0.771 \mathrm{~V})\end{array}$ & $0.618 \mathrm{~V}$ to $1.315 \mathrm{~V}$ & $0.015 \mathrm{uW}$ & 53.70usec \\
\hline TIQ & $0.59 \mathrm{~V}$ to $0.930 \mathrm{~V}$ & $6.046 \mathrm{uW}$ & 28.03usec \\
\hline ITIQ & $0.59 \mathrm{~V}$ to $0.930 \mathrm{~V}$ & $6.042 \mathrm{uW}$ & 28.02usec \\
\hline
\end{tabular}

Design of Encoder: Encoder is the digital part of Flash ADC which converts thermometer coded input to its equivalent digital output. For 3-bit Flash ADC the outputs of the corresponding thermometer codes are as indicated in the truth table as given in Table 2. The encoder is designed with following equations.

Table 2. Thermometer to Binary Code

\begin{tabular}{|l|c|c|c|}
\hline $\begin{array}{l}\text { Thermometer } \\
\text { code }\end{array}$ & $\mathrm{y} 2$ & $\mathrm{y} \mathbf{1}$ & $\mathrm{y} \mathbf{0}$ \\
\hline 1111111 & 0 & 0 & 0 \\
\hline 1111110 & 0 & 0 & 1 \\
\hline 1111100 & 0 & 1 & 0 \\
\hline 1111000 & 0 & 1 & 1 \\
\hline 1110000 & 1 & 0 & 0 \\
\hline 1100000 & 1 & 0 & 1 \\
\hline 1000000 & 1 & 1 & 0 \\
\hline 0000000 & 1 & 1 & 1 \\
\hline
\end{tabular}

In (Budanov, D. O.et al., 2018) proposed "high speed encoders" A high speed ROM encoder is designed in cadence tool with $180 \mathrm{~nm}$ technology. Fig. 7 depicts the schematic diagram of ROM encoder. ROM encoders are faster and their delay is independent of the resolution. Where as in case of Wallace tree, Fat tree, and multiplexer based encoders the delay depends number of input bits. To achieve faster conversion irrespective of number of inputs ROM encoder is preferred. The circuit converts thermometer code to digital code with very high speed. The high speed ROM encoder is designed with following equations 2 to 4 . Where y2, y1 and y0 are the outputs of the encoder and c1 to c7 are comparator outputs.

$$
\begin{aligned}
y 2 & =\overline{c 4} \\
y 2 & =\overline{c 2+\overline{c 4} c 6} \\
y 0 & =\overline{c 7}+\overline{c 1} c 2+\overline{c 3} c 4+\overline{c 5} c 6
\end{aligned}
$$

We use two ROM encoders in this design as with TIQ we use normal encoder but in single inverter based we get complemented outputs hence ROM encoder designed for complemented comparator output is used with single inverter based circuits.

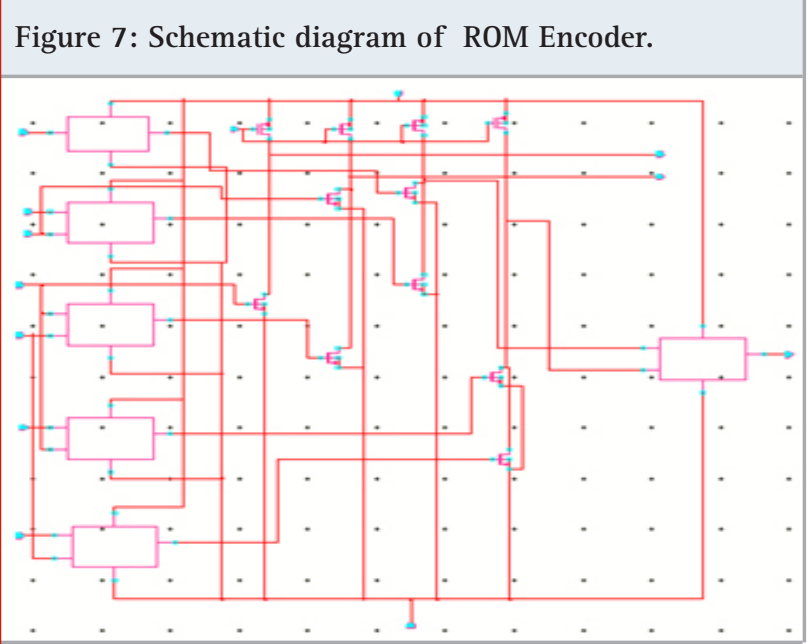

RESULTS

The inverter based comparators are designed and simulated cadence design tool with $180 \mathrm{~nm}$ technological library. The values of ' $W$ ' and ' $L$ ' are chosen by observing 
the results of parametric analysis. The simulated outputs of circuits are depicted from Fig. 7 to Fig. 12.

Figure 9: Transient response of ITIQ based comparator block

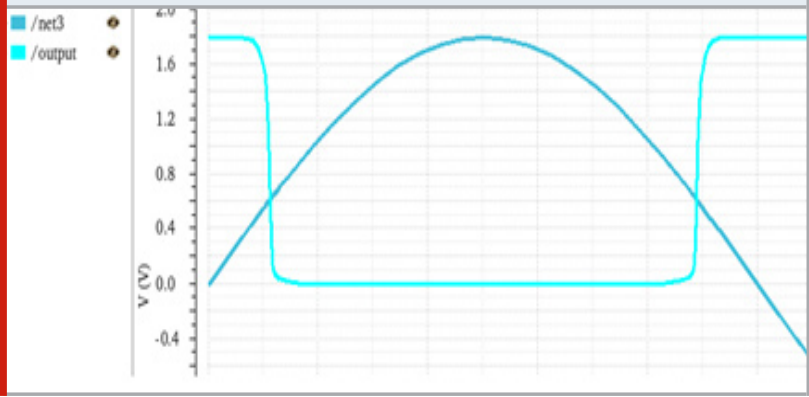

Figure 10: Transient response of Single inverter based comparator block.

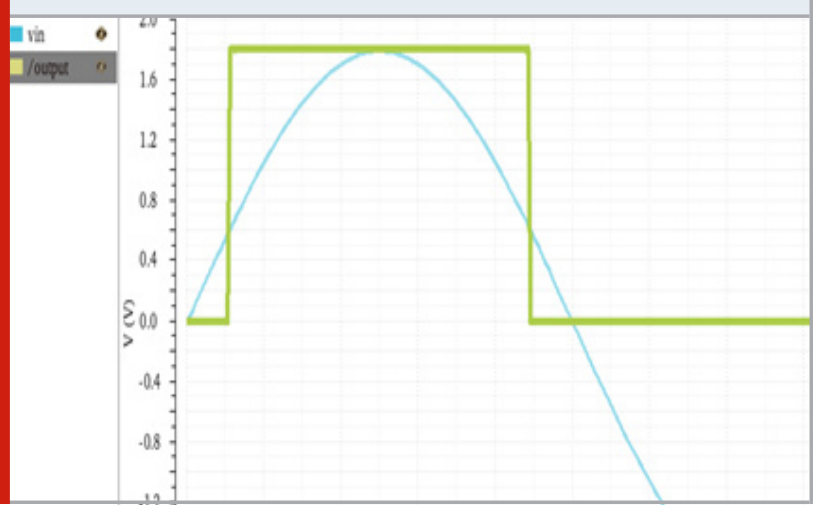

Table 3. Simulation results of Flash ADC designed with 180nm Technology.

\begin{tabular}{|l|c|c|}
\hline Flash ADC & $\begin{array}{l}\text { Avg. Power } \\
\text { Dissipation }\end{array}$ & Delay \\
\hline $\begin{array}{l}\text { Single inverter } \\
\text { comparator FADC }\end{array}$ & $554.06 \mathrm{uW}$ & $73.12 \mathrm{usec}$ \\
\hline $\begin{array}{l}\text { Single Inverter with } \\
\text { ref. Voltage } \\
\text { comparator FADC }\end{array}$ & $459.8 \mathrm{uW}$ & $2.832 \mathrm{nsec}$ \\
\hline TIQ FADC & $298.9 \mathrm{uW}$ & $73.16 \mathrm{usec}$ \\
\hline
\end{tabular}

The simulation results in Table 3 shows that the power dissipation is less in TIQ comparator based Flash ADC circuits than single inverter circuits. The results also show single inverter with reference voltage Flash ADC is faster among inverter based Flash ADC circuits.

\section{CONCLUSION}

The inverter based comparator circuits are designed using cadence analog design tool for 180nm technology. The simulation results show that as reference voltage is applied at the source of nmos transistor, power
Figure 11: Transient response of TIQ based comparator block

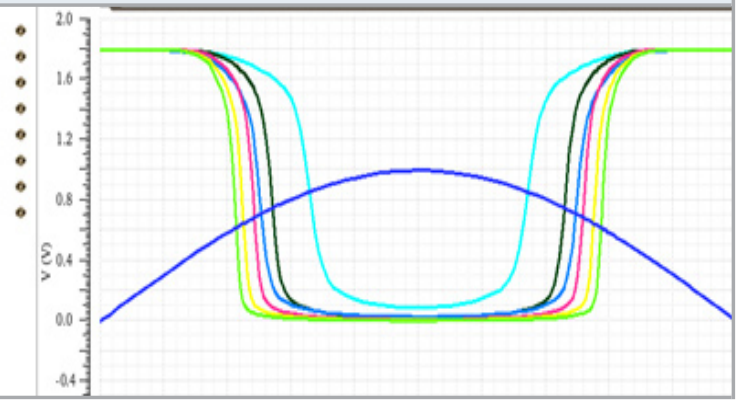

Figure 12: Transient response of designed 8:3 ROM Encoder

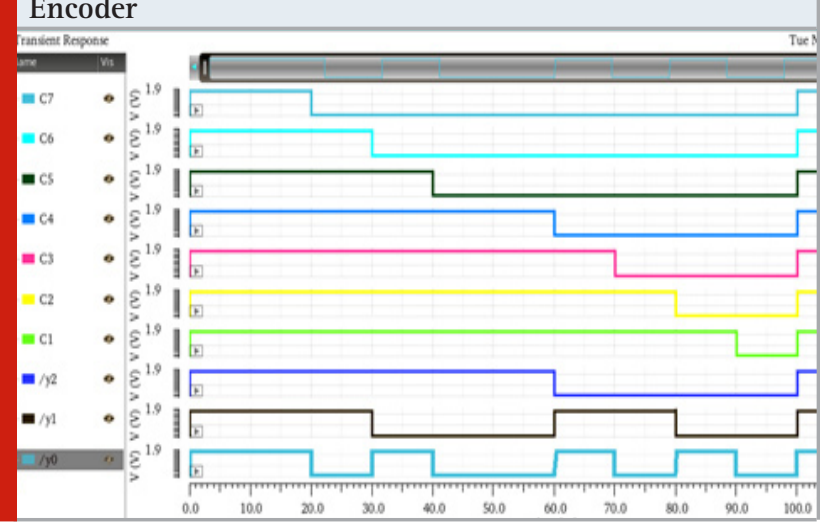

Figure 13: Output waveforms of 3-bit TIQ based flash $\mathrm{ADC}$ at $10 \mathrm{MHz}$.

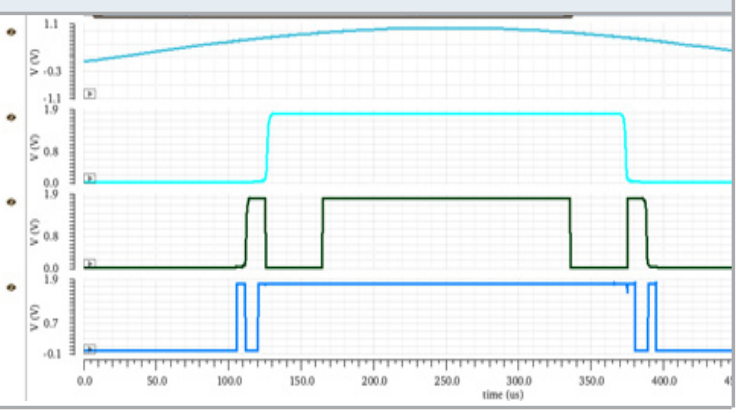

consumption is significantly reduced however leading to more latency. As reference voltages applied are different, the delay and power consumption, vary with reference voltages. 3-bit Flash ADC circuits are designed with different inverter based circuits. The results show that power consumption is considerably reduced by using TIQ comparators. The results also show that whenever speed is the criteria than single inverter based Flash ADC is the better choice.

\section{REFERENCES}

Budanov, D.O., Pilipko, M.M. and Morozov, D.V., (2018) January. Encoders for flash analog-to-digital converters. In 2018 IEEE Conference of Russian Young Researchers in Electrical and Electronic Engineering (EIConRus) (pp. 
173-177) IEEE.

Gupta, Y., Garg, L., Khandelwal, S., Gupta, S., Jain, S. and Saini, S., (2014) May. A 4-bit, 3.2 GSPS flash analog to digital converter with a new multiplexer based encoder. In 2014 11th International Conference on Electrical Engineering/Electronics, Computer, Telecommunications and Information Technology (ECTI-CON) (pp. 1-6) IEEE.

Hosur, K.N., Attimarad, G.V. and Kittur, H.M., (2015) December. Design of 4 bit flash ADC using TMCC \& NOR ROM encoder in 90nm CMOS technology. In 2015 International Conference on Trends in Automation, Communications and Computing Technology (I-TACT15) (pp. 1-6) IEEE. Conference on Inventive Systems and Control (ICISC) (pp. 742-747) IEEE.

Kalyani, N. and Monica, M., (2018) January. Design and analysis of high speed and low power 6-bit flash ADC. In 2018 2nd International.

Kar, A., Majumder, A., Mondal, A.J. and Mishra, N., (2015) January. Design of ultra low power flash ADC using TMCC \& bit referenced encoder in 180nm technology. In 2015 International Conference on VLSI Systems, Architecture, Technology and Applications (VLSI-SATA) (pp. 1-6) IEEE.

Kulkarni, M., Sridhar, V. and Kulkarni, G.H., (2010) December. 4-bit flash analog to digital converter design using CMOS-LTE Comparator. In 2010 IEEE Asia Pacific Conference on Circuits and Systems (pp. 772-775) IEEE.

Lavania, Y., Varghese, G.T. and Mahapatra, K.K., (2013) January. An ultra low power encoder for 5 bit flash ADC. In 2013 International Conference on Emerging Trends in VLSI, Embedded System, Nano Electronics and Telecommunication System (ICEVENT) (pp. 1-5) IEEE.

Madankar, A., Patil, M. and Chakole, V., 2015, January. Estimation of static and Dynamic characteristics for 4-Bit Flash ADC. In 2015 International Conference on Pervasive Computing (ICPC) (pp. 1-6). IEEE.

Perumal, I., Perumal, J. and Yuvaraj, V., (2009) October. Design of analog to digital converter using CMOS logic. In 2009 International Conference on Advances in Recent Technologies in Communication and Computing (pp. 74-76) IEEE.

Prathiba, G., Santhi, M. and Ahilan, A., (2018) Design and implementation of reliable flash ADC for microwave applications. Microelectronics Reliability, 88, pp.9197.

Yoo, J., Choi, K. and Lee, D., (2003) Comparator generation and selection for highly linear CMOS flash analog-to-digital converter. Analog Integrated Circuits and Signal Processing, 35(2-3), pp.179-187. 